\title{
Corrigendum: Endocytosed BCRs sequentially regulate MAPK and Akt signaling pathways from intracellular compartments
}

\author{
Akanksha Chaturvedi, Rebecca Martz, David Dorward, Michael Waisberg \& Susan K Pierce \\ Nat. Immunol. 12, 1119-1126 (2011); published online 2 October 2011; corrected after print 10 November 2011
}

In the version of this article initially published, the authors refer to the phosphorylation of Lyn observed after crosslinking of the B cell antigen receptor (BCR) as indicating activation of Lyn. However, the antibody to phosphorylated Lyn used (Cell Signaling Technologies catalog number 2731) recognizes Lyn phosphorylated at Tyr507, the negative regulatory site, and thus recognizes the inactive form of Lyn. Antibodies for the detection of the active form of Lyn (phosphorylated at Tyr396) are not available at present. However, the authors conclude that after crosslinking of the BCR, active Lyn must be in dynamic equilibrium with inactive Lyn at BCR clusters, as active Lyn is required for the observed phosphorylation of downstream kinases. They state that the following overall conclusions of the paper remain unchanged: BCR signaling is initiated at the plasma membrane and continues as the BCR traffics into the cell with sequential phosphorylation of kinases, and endocytosis is essential to regulate the outcome of signal. Nonetheless, they would like to correct this so readers are not misled about the activation state of Lyn observed in their studies. The error has been corrected in the HTML and PDF versions of the article.

\section{Corrigendum: The helicase DDX41 senses intracellular DNA mediated by the adaptor STING in dendritic cells}

\author{
Zhiqiang Zhang, Bin Yuan, Musheng Bao, Ning Lu, Taeil Kim \& Yong-Jun Liu \\ Nat. Immunol. 12, 959-965 (2011); published online 4 September 2011; corrected after print 17 November 2011.
}

In the version of this article initially published, the sensor DDX41 was suggested to recognize Z-form DNA in addition to conventional B-form DNA. Although GC-rich DNA has the potential to adopt a left-handed Z-DNA conformation under conditions of high salt or ethanol, whether the GC-rich oligonucleotides used the original study actually adopted the Z-DNA conformation remains uncertain. Therefore, the article has been corrected throughout to reflect whether poly $(\mathrm{dA}: \mathrm{dT})$ or poly $(\mathrm{dG}: \mathrm{dC})$ was used to stimulate cells. The errors have been corrected in the HTML and PDF versions of the article.

\section{Corrigendum: STING-dependent signaling}

Glen N Barber

Nat. Immunol. 12, 929-930 (2011); published online 20 September 2011; corrected after print 17 November 2011.

In the version of this News \& Views initially published, the sensor DDX41 was reported to recognize Z-form DNA in addition to conventional B-form DNA. This finding remains inconclusive as it was not shown that the GC-rich oligonucleotides used the original study actually adopted the Z-DNA conformation. The error has been corrected in the HTML and PDF versions of the article. 